\title{
Energy and Information
}

\author{
Borza Paul Nicolae ${ }^{1}$, Sanduleac Mihai², \\ Musat Ana Maria ${ }^{1}$ and Carp Marius Catalin ${ }^{1}$ \\ ${ }^{1}$ Transilvania University of Brasov, ${ }^{2} E C R O S R L$ \\ Romania
}

\section{Introduction}

In thermodynamics a native link between energy and information is defined by using the entropy as a measure of the microscopic states which reflect the total energy of the system. In electrical domain the distinction between power systems and information systems is more clearly defined.

Energy represents the capacity of changing the state of a system or performing work. There are multiple forms of energy but all of them respect the conservation law, a consequence of the fact that the laws of physics do not change over time (Noether's theorem). Thinking at the usage of the energy as engine of the processes transformations it is important to focus on this maximum efficiency having in mind all the transformation stages from generation, storage and conversion down to consumption. Nowadays, the processes optimization is an essential and complex research and technological challenge. The impact of this effort is essential for the whole human society and also for the environment.

The two essential terms, energy and information, are in general viewed as dichotomist entities. In fact, these terms are strongly related in both stationary and mobile power sources applications, in order to reach a high energy efficiency of the system.

"The whole is more than the sum of its parts is simply that constitutive characteristics are not explainable from the characteristics of the isolated parts. The characteristics of the complex, therefore, appear as news or emergent in case of General System Theory"-1950 (Bertalanffy, 1969). It can be affirmed that the information is represented by news. Signals offer the support for information and also express its energetic facet, mandatory to transfer information between two entities.

Neither the power sources nor the storage devices can cover with maximum efficiency all the requirements of the load. In order to optimize the energy flow, the actual researches are focused on developing hybrid power supplies composed of different sources and storage elements, such as: batteries, supercapacitors and fuel cells. All the components are interconnected by a power distribution grid intelligently controlled by switching devices or inverters (Borza, 2007). 


\section{Energy}

Starting from the biological paradigm, the reality of energetic processes can be observed at the cell level, in the context of living organisms. Thus, every cell has as energetic resources three different types of "fuels". First, ATP (adenosine triphosphate), offers the fast response and also, for a short period of time, the energy of the cell by oxidation of its macro energetic chemical links. The second, ADP (adenosine diphosphate), offers by oxidation the energy necessary for the cell on medium term, maximum several minutes. For the long term energy requirements, a transportation phenomenon using the blood flow assures the access of the glycogen at the cell level where it will be oxidized thus offering the necessary power for the living body. This gives the third type of "fueling" the biological system.

Going forward by analogy, it is possible to conceive a combined energy source that includes several types of generators and storage elements such as: supercapacitors able to assure the fast release response (below 1-2 second) as the ATP, batteries for mean term response (several minutes) as ADP and fuel cells for supporting the long term response (depending on the tank of hydrogen) as glycogen in living body.

Supercapacitors are reversible storage devices suitable for peak power requirements of the load and fast response in time because they store energy as separate charge inside the device, involving no major chemical reactions (Conway, 1999; Gualous et al., 2003). For these devices the bidirectional power transfer is assured with a high efficiency going up to $98 \%$.

In the case of the batteries (secondary types), the chemical energy contained in their active materials are converted into electric energy by electrochemical redox reactions. The majority of these reactions are quasi-reversible, the materials of the batteries suffering a depletion and degradation, during their life. The batteries are very sensitive devices in relation to the type of demands and its variation during the time. Also, the depletion and degradation of the batteries' materials determine significant changes of their parameters, measured for example by "batteries state of health" (Pop et al., 2008) and reflected by the batteries' reliability. Thus, the predictability of the batterie' life time is difficult to be done. In general, these devices are very sensitive at load demands and their variation, at temperature and deep of discharge (DoD) (Linden \& Reddy, 2002) or at the complementary parameter State of Charge (Pop et al., 2008). Even respecting all these constraints the cyclability of devices is limited - function of the type of battery, from several hundred to several thousand cycles (Linden \& Reddy, 2002; Pop et al., 2008).

In case of the fuel cells, the generation process involves (i) a reversible chemical reactions that cannot be instantaneously activated and (ii) a degradation of charge separators in time which decreases the life time of devices. For these devices the main problem is related to the low energy density of hydrogen or its derivates liquid or solid components that are separated in order to generate the requested energy. More than that, the fuel cells have a long time constant, are able to provide the requested energy for long term, thus increasing the autonomy of the system. A raw image about the energy and power density offered by different power sources or storage devices is done by Ragone diagram (Flynn et al., 2004).

The reliability, the cyclability and the life time of the last two power sources described above is strongly affected by the specific functioning regimes if they are used individually. In order to eliminate these disadvantages, the present researches are focused on finding a compromise (but synergetic) solution by combining, sizing and optimal controlling the three above mentioned devices (Borza, 2007). 
A possible theoretical and simplified structure of the combined energy cell (CEC) is illustrated in Fig. 1. At the cell level the components depending on their time constant together with the local controller will be cascaded. The controller has two roles: (i) to manage the power flow between the components of the combined cell and (ii) to optimally integrate the cell into a grid of cells. Thus, based on the information transferred using the wire/wireless interface, based on the monitoring stages of the CEC and based on specific control strategies, the controller will find out the appropriate connectivity for the assembly of the switches. The cell controller will play two roles: (i) to monitor and supervise the internal power flow between the main components of the cell: fuel cell, battery and supercapacitor and (ii) to communicate with the adjacent similar cells in order to settle and command the appropriate switching strategy that will satisfy the load demands at the system level. In this sense, two types of connections are possible to be implemented: series connection (SB+\& SB-) and parallel connection (PB+ \& $\mathrm{PB}-$ ). Function of the external and internal parameters, a bypass switch can shortcut the whole cell in series connection, and by switching off the booth switchers -series and parallel - the cell can be completely insulated related to the cell array. The architecture of the CEC is illustrated in Fig. 1. This architecture douse not specify the additional components necessary to enssure a smoothing switching process. Such architecture involves the development of an important information processing stage in order to assure the close control for the array of combined energy cells. The main constraint necessary to be satisfied is the "real time" condition that suppose for the local controller to have an important calculation power in order to assure the stability and also smoothness of the commutation process.

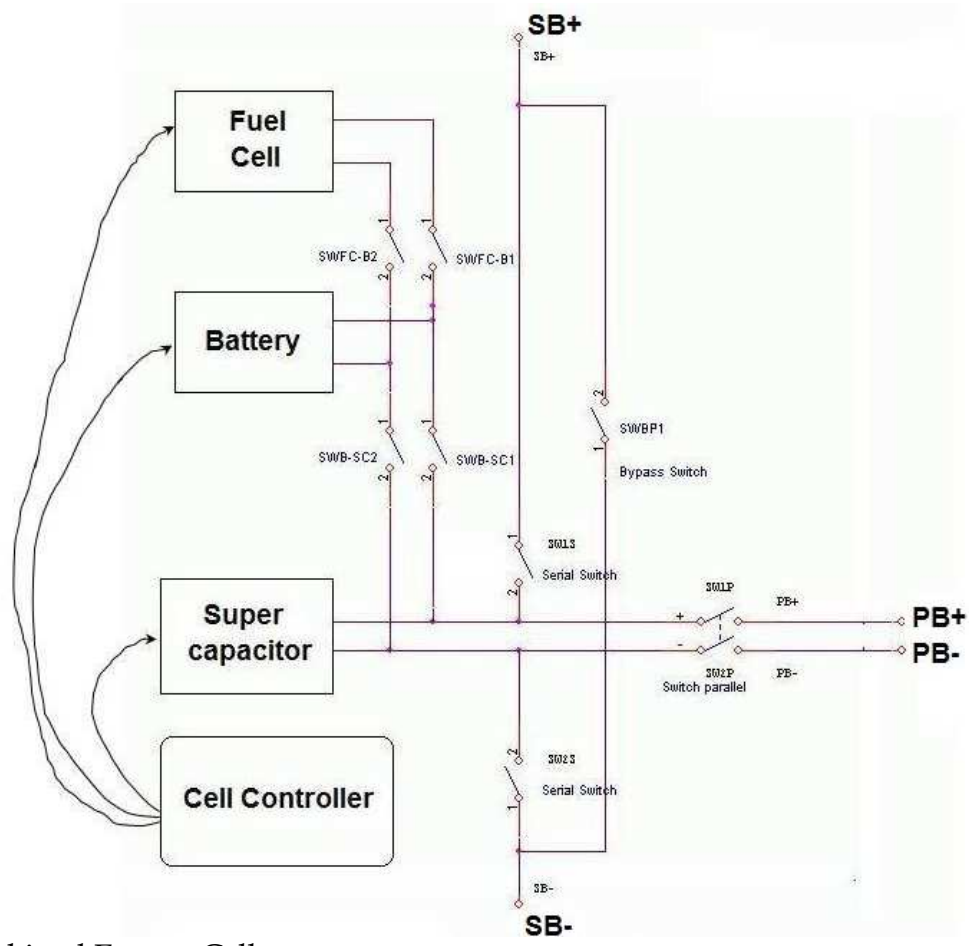

Fig. 1. Combined Energy Cell 
The system integration process supposes the development of a set of strategies and response patterns at the demands arrived from load side. The architectural approach is illustrated in Fig. 2.

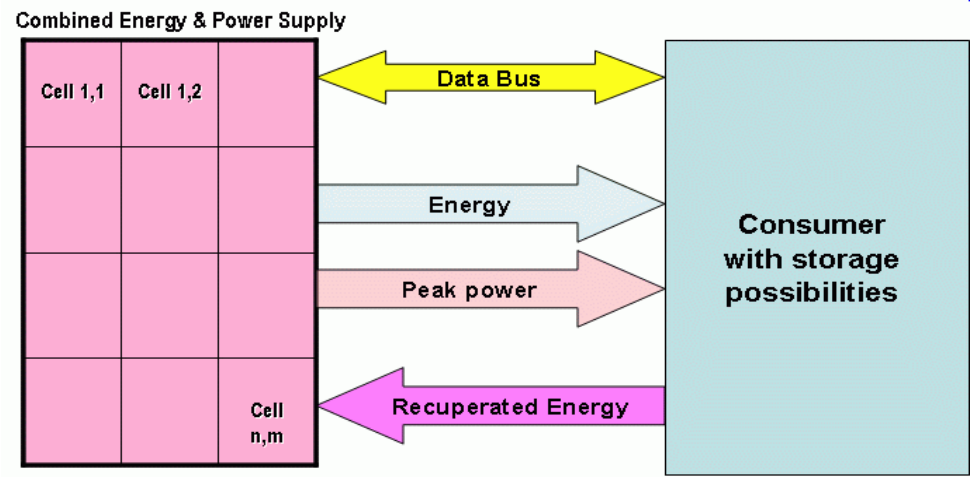

Fig. 2. Cell's array. Architectural approach

About this approach it is also important to observe that for the majority of the applications that request a storage element and an energy converter beside load, the redundant cells and also the complementary stages of these are mandatory in order to increase the energy efficiency. The sizing process of the combined energy cell is a complex process mandatory for providing the energy and power requirements with minimal costs. During the sizing design, the both sides of the problem, optimization generation and storing versus conversion and consumption, must be taken into consideration. We shall refer to all the characteristics of the device such as: the energy and power density, the topology, the granularity and redundancy of the power supplies and the energy storage devices; their life time, cyclability, and reliability. It is also necessary to take into account the eventually degradation of the energetic performances of the device.

Energy .... As Capacity to be provided
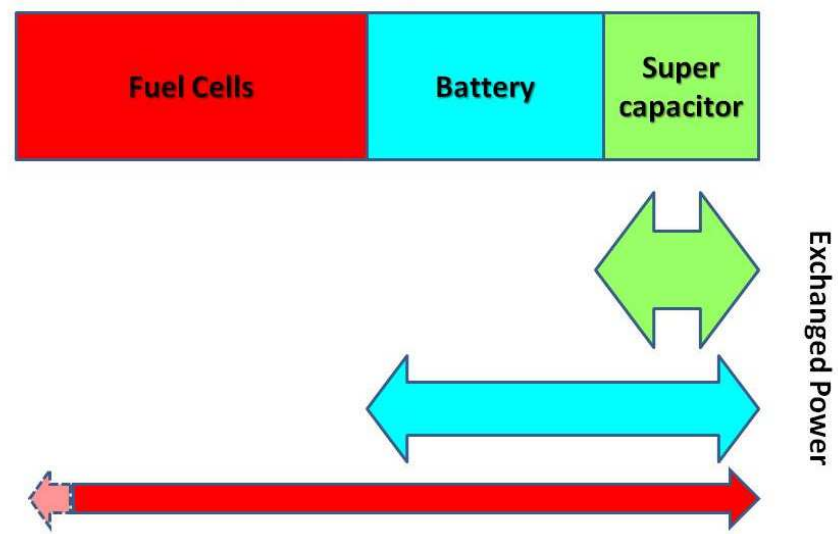

Fig. 3. Sizing process: energy vs. power 
On the other hand, the problem refers to the demands from the load side. The performances of the load depends in principal on the maximum and steady state power flow, the transport mission, the "driving style", the traffic, and so on. In is illustrated a simple modality of satisfying the variety in time of the power exchange capacity by using the supercapacitors as storage elements (rapid release storage elements). Thus, the basic voltage, speed, power or current regulators used for controlling the power flow are not enough. For increasing the efficiency, it is necessary to design an intermediate level (like a middle ware) in which, based on the dynamic settlements of the regulators, the system will adapt permanently his control strategies at the influence factors and demands. Even the simplest processes such the optimal braking process need a lot of measurements to proof the strongly link between the energy efficiency and the information necessary to assure the optimal control.

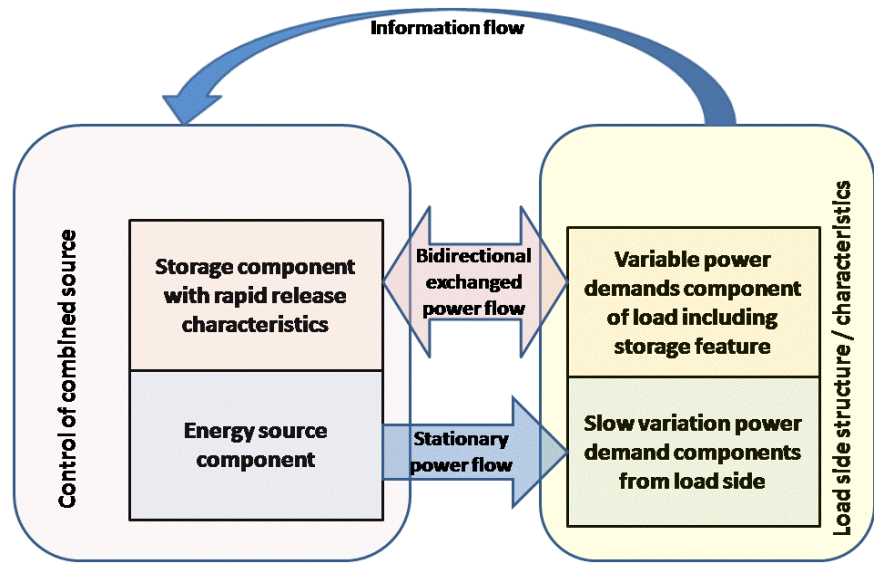

Fig. 4. Ideal architectural view of a recuperative braking system where all the variation of energy is exchanged with the supercapacitors elements

In order to give an example, the power limitations of the components integrated in the combined energy cell it can be considered. This is significant for fuel cells and also for batteries but allow a large exchange of power in case of the supercapacitors. More than that, only several types of fuel cells are able to provide or to absorb the energy, most of them being not reversible. A comparative image between energy and power density of the three types of devices is illustrated in Fig. 3.

For the sizing process, the steady state energy can be assured by the fuel cell, able to provide for long term energy with low power density. Instead, for high power density, the supercapacitors are mandatory to offer the power reserve in acceleration or deceleration processes (Fig. 3.). The performances of the batteries are placed between the above described devices. Also, such a structure with rapid release storage devices permits the regenerative braking implementation.

The parameters taken into account in case of supercapacitors are: nominal voltage $\mathrm{V}_{\mathrm{scN}}$, capacity $\mathrm{C}_{\mathrm{sc}}$, and the equivalent series resistance $\mathrm{R}_{\mathrm{ESR}}$. Thus the maximum released power can be provided by supercapacitor at the initial moment of discharge (1), respectively the 
maximum absorbed power appears at the moment when it starts to be charged the completely discharged supercapacitor.

$$
P_{\max }=U_{\max } \cdot I_{\max }=U_{\max } \cdot \frac{U_{\max }}{R_{E S R}}=\frac{U_{\max }^{2}}{R_{E S R}}=\frac{Q_{\max }^{2}}{C_{s c}^{2} \cdot R_{E S R}}=\tau_{s c} \cdot \frac{Q_{\max }^{2}}{C_{s c}}
$$

where $U_{\max }$ is the maximum voltage and $\tau_{s c}$ time constant of the supercapacitor. For the battery the maximum power is related to the series battery resistance and this parameter is dependent with temperature, state of charge (SoC) and state of health $(\mathrm{SoH}), I_{\max }(\vartheta, \mathrm{SoC}, \mathrm{SoH})$. Thus the maximum power (2) is done by:

$$
P_{\max }=U_{B a t} \cdot I_{\max }
$$

Unfortunately, during the commutation process for batteries the equivalent series resistance is variable from a high value to a steady state value. This is the consequence of chemical reactions "inertia" and generates an increasing of internal power consumption during commutation process. In general is better to respect an upper limit of discharge or charge current for battery and these values are directly related to the maximum power allowed by the device because the normal voltage variation on battery is not significant. The maximum power provided by the combined energy source is not enough to describe how their power is transmitted or absorbed to or respectively from the load. To have a realistic image it is necessary to consider the characteristic of the provided power by all the components integrated in the combined energy cell. Besides the features of these devices it is also necessary to consider their constraints related to the limits of current and voltage variation of the batteries and fuel cells in the domain of temperatures, cyclability and reliability. Thus, the control system will be able to reach a maximum efficiency only when it will consider the main influence factors, the characteristics and the constraints related to the combined energy cell's components.

The complexity of the control is directly related to the complexity brought by matching devices' characteristics that are dependent from a lot of the above mentioned factors, including time.

While studying the regenerative braking process, two important equations $(3,4)$ reveal the need to balance the electric energy stored on supercapacitor and the kinetic energy provided by the vehicle. In this process an important role is played by the adequate devices sizing function of application constraints and performances. Thus, in case of a vehicle it is suitable to recuperate the majority of the kinetic energy of the vehicle using regenerative braking. Thus, the two forms of energy kinetic (3) and electric (4) have to be balanced. unfortunately, during recuperative braking process the supercapacitor will be charged and thus the braking force will decrease in case when it exists only one combined energy cell. Also, this desire is in contradiction with the safety prescription related to the braking of vehicle maximum braking force and mandatory compliance of this with the driver intention (fidelity of braking control). 


$$
\begin{gathered}
W_{S C}=\frac{1}{2} C U^{2} \\
E_{k}=\frac{1}{2} m v^{2}
\end{gathered}
$$

If it is considered the possibility to use batteries as storage devices during recuperative braking process the efficiency depends on batteries' state of charge, temperature and maximum current recommended by the manufacture. The high peak currents on batteries can drastically reduce their life time. In order to eliminate these constraints, an intelligent control system able to predict the emergency braking situations and the current limit has to be implemented. Also, a modality to absorb the high peak currents is to switch the load of vehicle's motor in generator regime from one to the other discharged supercapacitors by the power electronics switching devices in order to maintain during braking process an important recuperative current that will be stored in the supercapacitors. Even so, at very low speed of the motor, a dynamic braking procedure (to spend the last part of kinetic energy of the vehicle on a power resistor) will be necessary to use in order to stop the motor. Another example that illustrates the idea of deep and discrete power flow control is the starting system realized for diesel hydraulic locomotive, where the adequate commutation between the different power generators and storage devices optimize and make more efficient the starting process of diesel engine (Carp et al., 2010). On classical vehicles, in order to start the internal combustion engine (ICE), an electric motor supplied in general from vehicle's batteries ICE shaft rotates. Synchronously, the board computer will supply with fuel and eventually will transmit the ignition command to each cylinder, initiating the fuel explosion and finally starting the engine. The process is relatively fast has to transfer an important amount of energy to the engine in short time in order to reach the starting conditions for the ICE. In order to assure a reliable starting process of the engine on a large temperature domain, in general, the capacity of the batteries placed on vehicles is over dimensioned. Using the combination of batteries and supercapacitors, the system is shown in Fig. 5 where $B$ represents the locomotives batteries (150Ah), $K_{1}, K_{2}, K_{3}$ are the power switching devices, SSC is the assembly of supercapacitors $(210 \mathrm{KJ} / 110 \mathrm{~V})$ stacked with aqueous electrolyte and $\mathrm{M} / \mathrm{G}$ is the staring electric motor $(25 \mathrm{KW}), \mathrm{R}_{\mathrm{L}}$ is the limitation resistor of charging current and ICE is the diesel engine of locomotive (920KW).

The information related the starting process parameters will be provided to the starting computer that will control the provided energy by the batteries during the initially phase. In the initial phase, under the starting computer control and using $\mathrm{K}_{3}$ as switching device, the supercapacitor SSC will be charged. The energy level will be in accordance with the necessary amount of energy that is quickly transferred, using the starting motor to the ICE's shaft. This action will generate from the first stroke, the ignition of fuel inside engine's cylinder. At locomotive driver initiative $\mathrm{K}_{1}$ switch will be closed thus the energy stored on supercapacitor will be transferred to the starting motor. Because the starting process need more time in the third phase $\mathrm{K}_{2}$ will be switched on thus blocking $\mathrm{K}_{1}$ and assuring the finalization of the diesel engine starting process. 


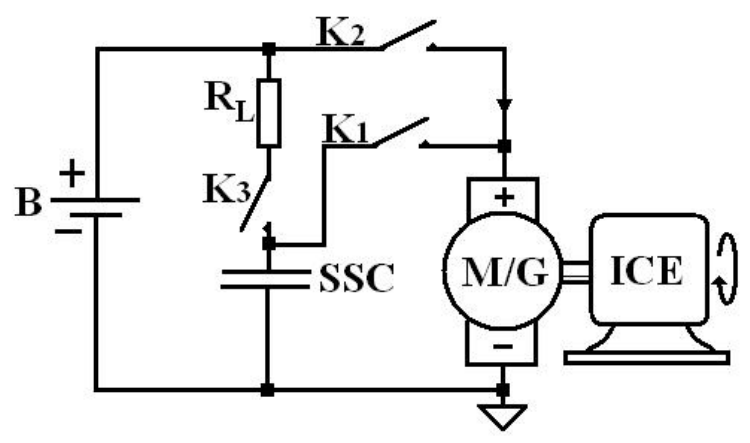

Fig. 5. Structural scheme of the staring system of diesel hydraulic locomotive LDH1250 endowed with supercapacitors

The information plays the essential role in this case of assuring the optimization of the power flow transfer between the supplies (batteries) to the ICE shaft. By scheduling the power flow transfer between batteries, supercapacitors and again batteries, the rapid release storage device (SSC) will be prepared to quickly transfer the necessary amount of energy to the ICE via the staring motor. In order to stabilize the ICE regime, the batteries will provide the additional amount of energy during the final phase of starting process (Carp et al., 2010). Monitoring with the board computer the power flow transfer in conjunction with the evolution of ICE's starting process, the switching time between $K_{1}$ and $K_{2}$ is established in order to assure the optimal balancing power between the transferred energy from supercapacitors and batteries. It is also assured the batteries' protection against the over current typical for such processes and the maximum energy efficiency for the whole process. In Fig. 6 are shown the power flows from the starting process.

\section{Thermal disipation}
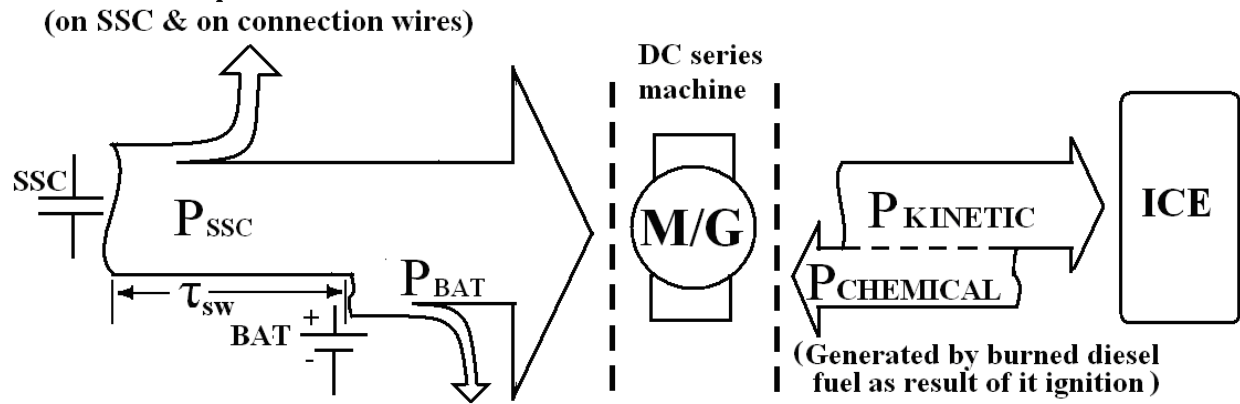

Thermal disipation

(internal on batteries \& on connection wires)

Fig. 6. Power flow distribution during starting process of locomotive's diesel engine 
This example proof the importance of information from process used for controlling the power flows using two kinds of methods: the scheduling of power flow transfer and the detection of power flow provided by the internal combustion engine during starting process. The variation of the main electric parameter: V_Scc voltage on supercapacitor, V_Bat voltage on batteries, V_Mot voltage at the terminals of starting motor, I_Mot current on the staring motor and RPM_Fvc the rotation speed variation on starting motor shaft, on the starting circuit are illustrated in . The voltage on supercapacitor is bigger than battery's voltage because this is charged during the last functioning period of locomotive when the generator provides the overvoltage necessary for charging the batteries.

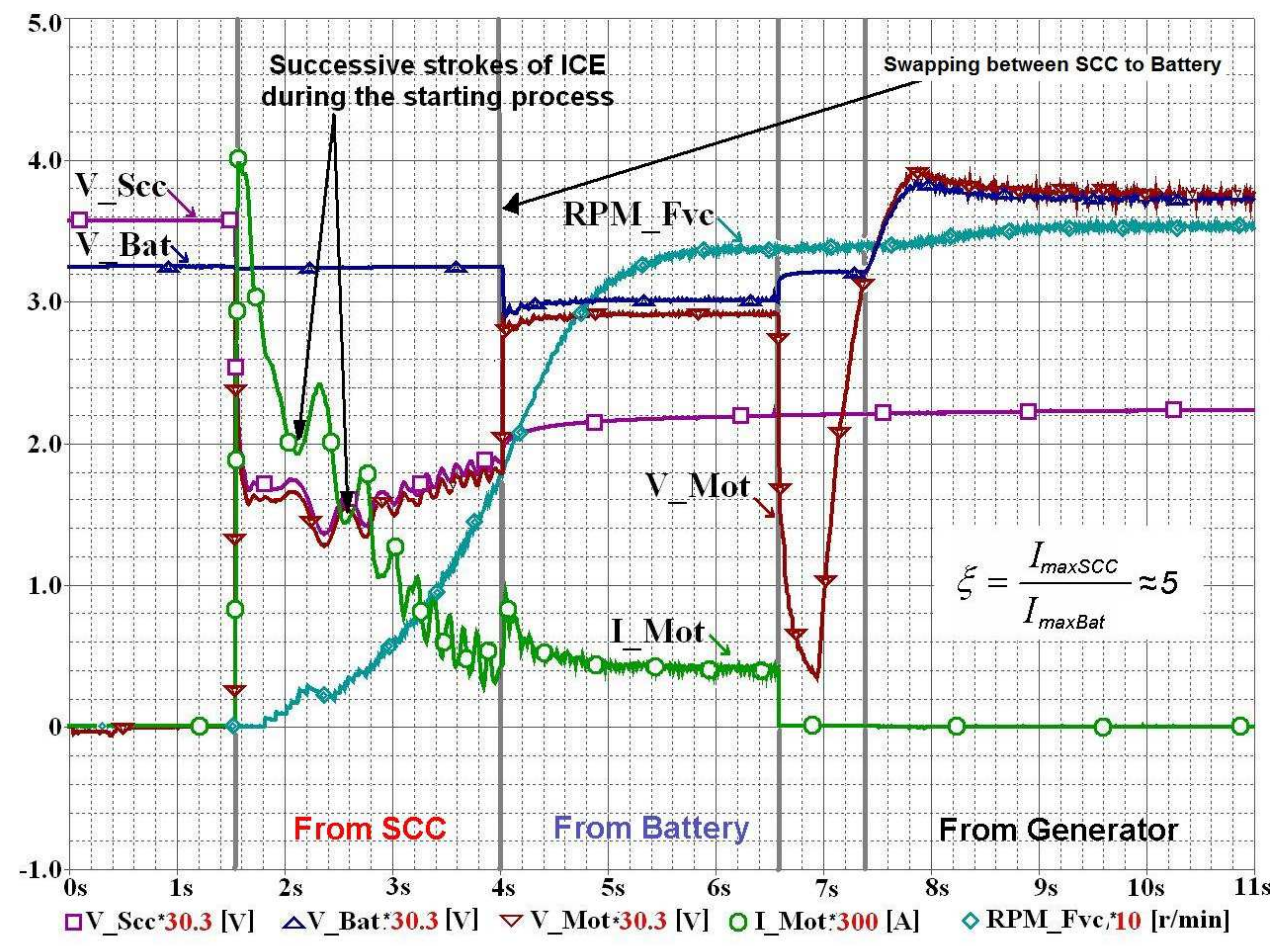

Time

Fig. 7. Diagrams of voltage on supercapacitor, batteries, motor, current and rotation speed of the starting motor in time

The swapping between supercapacitor to batteries on electric circuit of starting motor will be controlled by the board computer. An important goal of the controlling system is to maximize the ratio between the current provided from supercapacitors and batteries $\xi$. Another goal, also essential, consisting in optimization of fuel ignition process is achieved by the appropriate sizing of the system components, supercapacitors and batteries that assure the adequate and rapid release power flow from electrical side to the diesel engine.

Finally it is interesting to mention that the implementation of the presented system has generated several savings consisting in: halving of batteries' capacity on locomotive in 
parallel with the improvement of success rate of ICE starting process, reduction of fuel consumption on the locomotive with more than 301/day, and an estimated batteries' life time increase with more than $70 \%$.

\section{Cloud computers acting as Virtual Power Plants - a way towards energy smart cloud-computing in a Smart Grid environment}

\subsection{Current status}

Today developments of computers migrate to new concepts, where cloud computer are more and more used and studied. One characteristic of cloud computers is the fact that their resources are aggregated and virtualized, in order to increase the computation capacity, the reliability of computation system and also to assure the reserve and other key characteristics that are monitored and controlled.

It is well known that the produced electric energy has to be balanced with its consumption continuously. For this reason it is necessary all the time to monitor the generation and the consumption of energy. Nowadays this aspect becomes more important in case of energy produced using renewable energy sources (RES) where the generation is dependent of natural factors, the generators are spread on large areas and also its nominal power is not very high.

Energy efficiency is a new focus in cloud computers. The energy consumption of the cloud computers should be controlled thus to optimize the energy consumption for a specific computing effort. Techniques like shut-down, hibernate or sending in different low power stages are current areas of analysis in order to achieve more "green" computation (or more exactly to decrease the KWh/TFlop). In (Taft, 2009) there are some relevant declarations with ask for improvement of energy consumption: "Future data centers will be dominated by energy cost, with half of that being spent on coolant" and "We cannot afford anymore to design computer systems based on the criterion of computational speed and performance alone".

\subsection{Using Virtual Power Plants for maximizing cloud computers benefits}

The best solution may still need a broader approach. When we speak about energy reduction as main scope we can also go further and speak about scope functions like energy cost per TeraFlop or even to $\mathrm{CO}_{2}$ kilograms equivalent to provide a Tflop of computation.

When we speak about energy, our today's scope is either a) focused on reducing $\mathrm{kWh} / \mathrm{TFlop}$ to Euro / Tflop - which is a manager oriented approach looking for operational costs, or b) reducing the $\mathrm{kg}$ of $\mathrm{CO}_{2} / \mathrm{Tflop}$ - which is an ecologist oriented approach looking at impact on the environment. However the picture it is not complete yet: when we look for energy, Euro or $\mathrm{CO} 2$ reduction, we need to look in parallel to a) the cloud computer entity which aggregates the power of the computer components and b) the energy consumed by each computer which can be aggregated in a virtual distributed consumer, connected to the power network in various places, many times supplied by different suppliers, connected to different distributors and acting simultaneously in different countries. The power consumed by the different computers in the cloud has also two aspects: a traditional energy aspect and an ancillary services aspect. The last one deals with the fact that energy consumption in a cloud can be influenced by stopping or powering again some of the computers, which is a flexibility needed in special situations by the transmission system operator (TSO), in order 
to avoid critical regimes and its use is seen by TSO as a service provided to him, in order to maintain stability of the system. This last aspect is a power system oriented approach, looking on the impact of the consumption to the power system operation.

A particular interest in energy networks is the SmartGrids technological platform endorsed by European Union, where the existing status-quo of the power networks is boosted towards new horizons by implementing active networks, distributed generation, flexible energy and ancillary services markets.

As this SmartGrids new approach is still at the beginning, one precursor of the concept already proves the efficiency in the early phases of this new wave: the technology of the so called Virtual Power Plants (VPPs). This VPP technology allows a better utilization of many small distributed resources (FENIX, 2009), in terms of energy contracts (better price for the energy) and of ancillary services (possibility to gain more money based on flexible production or consumption, within power control bands which can be used on request, based on the needs of the network).

As previously mentioned, cloud computers are energy consumers which have the possibility to modulate their consumption, based on their actual tasks. For instance, if only $60 \%$ of the general computing power is used, then a part of the physical machines can be shut down, thus reducing the overall power consumption of the cloud computer. An important feature is related to the energy consumption which can be controlled by using specialized software dedicated to cloud computer.

With this functionality, cloud computers can act as flexible consumers.

An important aspect is that in the VPP concept, not only generators are considered resources, but also the flexible consumers, as the cloud computer can be. Moreover, the cloud computer not only is a possible flexible consumer, but his consumption is already aggregated and controlled by the cloud computer software, so it acts like a Virtual PowerPlant (VPP).

Considering these aspects, a new dimension of the cloud computers can be expressed:

- The cloud computer can aggregate its distributed consumption in an aggregated virtual consumer with flexible behaviour;

- The virtual flexible consumer can act as a Virtual Power Plant

- The aggregated energy of the new VPP can be obtained from the energy market, through a VPP energy purchase technique;

- In order to act as a flexible consumer, the cloud computer can divide its tasks in:

- mandatory or uninterruptible tasks, those which have to be provided independently of external factors of the energy market

- energy low price oriented tasks, which will run when energy spot market price is low, for instance when energy is highly available in the network midnight period or when wind power production is exceeding the normal consumption, so the prices are going low in order to encourage more consumption

- Low $\mathrm{CO}_{2}$ related tasks, where tasks and a part of the computers in the cloud are running only when low $\mathrm{CO}_{2}$ production is present on the energy market (during high wind or high hydro production, during night production with nuclear powers etc.)

$\circ$ a combination of the above 
- The cloud computer can reduce temporary the whole power by the request of the energy network, providing so-called tertiary (power) reserve, which is a paid ancillary service even inside a traditional power network (not only in the new SmartGrids concept).

With this approach, a combination between the two systems (cloud computers and power networks) brings new dimensions and new potential business models and income:

- Cloud asset owner gets income by selling most of the computational power as it does now;

- The asset owner can sell a part of its computational power, scheduled to run in cheap energy and low $\mathrm{CO}_{2}$ periods

- The owner can get additional money by accepting to be shut down or to reduce power on special request of the network - when a special situation occurs - and to be paid for the availability of this action. The reaction time for such reduction can be between 5 and 15 minutes and the period of reduction can last from 15 minutes up to two hours

- Cooling of the local supercomputers provides accumulation characteristics and the energy usage for coldness accumulation can be synchronized with energy market signals, thus optimizing consumption costs in a certain amount.

A concept which considers both systems by using Virtual Power Plant concept is presented in Fig. 8. In this figure, all power consumptions of a cloud computer are aggregated in a VPP.

Through a VPP agent the cloud accesses mechanisms of the energy market and gets:

a) Favourable energy prices,

b) Additional income due to load flexibility on demand (by performing ancillary services on request) and

c) Potential additional income due to synchronization of consumption with green energy production.

Computer systems and energy systems are integrated systems which usually work in parallel, without other interaction that one-way energy supply. The nature shows us that such parallel networks need more complex interaction in order to achieve overall performance.

It is not the first time that human beings are excellent models of inspiration for solving engineering problems (Brandic, 2009). For instance, nervous system in a living being can be considered in a way similar with the cloud computer. The power network is another independent system, which can be considered similar with the sanguine system. In human being the two systems interact and optimize their functionality. The new approach for cloud computers interaction with the power system can be considered a similar situation in the engineering field. 


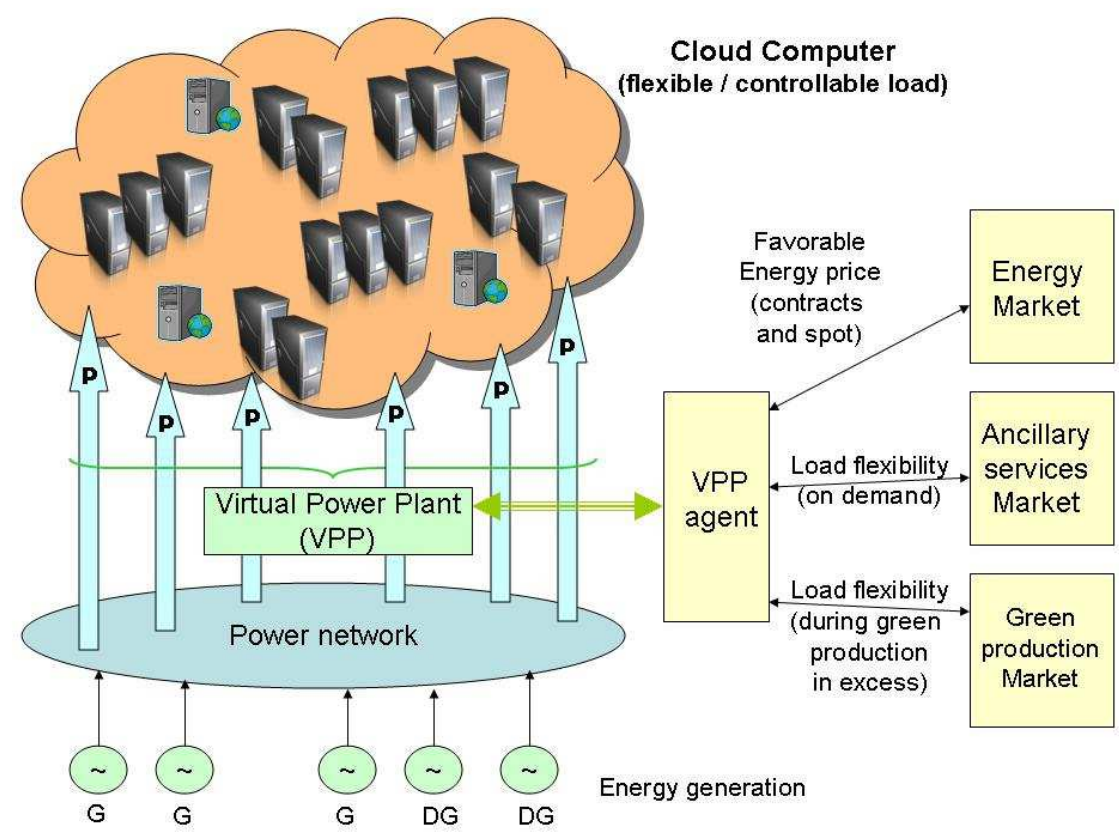

Fig. 8. Conceptual approach based on Virtual Power Plant model

In this way, some features of both system become synergic: on one hand the forecast that could be provided by the cloud computer offer a confident image about the consumption demands and on the other hand the power network allow to spend during the low demand period the over energy production as cool inside data centers (thermal storage) of energy allowing a better performance for computers, with low exploitation costs.

We can imagine for instance that the peak of a supercomputer / cloud computer is controlled to be reached such tasks. More than that, some tasks are computed only outside the peak power of the network. As an example, this can happen when for instance the peak power of the power system is between 19 and 20 hour of the Central European hour (UTC+1h). In this period the local supercomputer can temporary reduce its computational and energy power at $40 \%$ of the nominal power.

After the energy peak is over, the cluster can go back to full computational and energy consumption power. In this period, another part of the cloud reduces its power due to peak energy power in other power systems (for instance at UTC+0h time).

With such setup we obtained a reduction of consumption with computers of $60 \%$ in each local power systems but if the cloud is spread equally on 3 time slots, then the total computational power has been reduced with only $20 \%$, so we got a small reduction in the cloud computational power but with a good impact in each local power system, with potential positive impact on Euro/Tflop and/or on $\mathrm{kg}$ of $\mathrm{CO}_{2} / \mathrm{Tflop}$.

The projection of this mechanism on three time zones of Europe is shown in Fig. 9. 


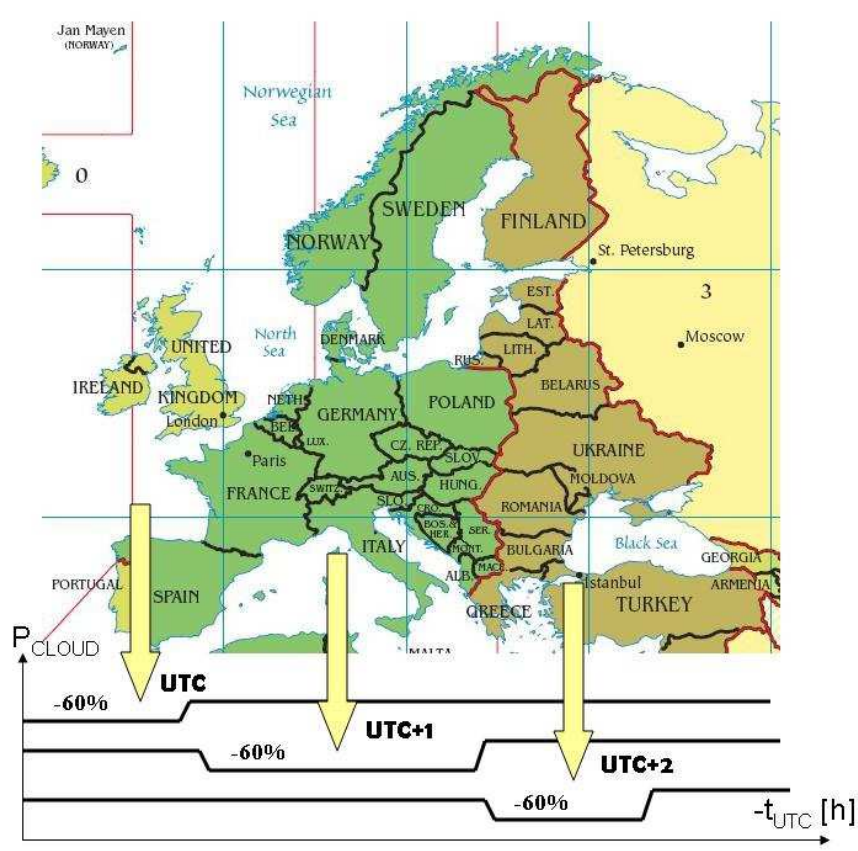

Fig. 9. Time-zones and their role in consumption of electric energy produced

We can bear the following conclusions: cloud computers system and energy network systems can interact and produce synergies:

a) the cloud computers can get smaller energy bills or even can provide services to the power network, thus maximizing their benefits;

b) the power system has a better knowledge about the aggregated consumption and can rely on load control services if these are necessary and agreed by the parts.

In addition, energy storage behaviour might be also possible to be implemented, by overcooling the cloud during favourable time periods.

The model is comparable with living systems, which have also multiple interactions between the various subsystems - thus achieving high performance, economic operation and higher reliability.

\section{GreenIT}

Nodaway approximate $10 \%$ of total energy production over the world is spent by the IT industry and personal computers. Lately, reducing the IT energy consumption is an important aim that needs to be assessed. A prospective image about the possible evolution scenarios for electricity use by data centers existing in United States show annual savings in 2011 of approximately 23 to 74 billion KWh compared with the trend detected in 2007 by US Environmental Protection Agency (EPA, 2007). Unfortunately, in present, there are no efficient implementations for measuring, monitoring and interpreting the data flow inside of 
a system. Looking at the overall energy consumption in case of data centers approximately $50 \%$ of energy are spent for cooling, chilling, heating, ventilation and air conditioning (HVAC), other $48-50 \%$ are spent by the IT equipment and only several percents for the ancillary services (Schulz, 2009). A detailed image is offered in (Schulz, 2009) and approximately $50 \%$ of the IT power consumption is dedicated for servers. Unfortunately only relatively a low part $(25 \%)$ of total computation power of the data centers is used by their servers. Green IT has to come with both hardware and software implementation able to assure together accurate management boards for optimizing the energy consumption and for increasing the efficiency of the all ensemble. The Green IT technologies has appeared as a necessity to manage the complexity of the optimization problem of computing needs and putting this in accordance with the energy efficiency aims and the principles of sustainability of related those activities. In Fig. 10. are presented the factors involved by this technology.

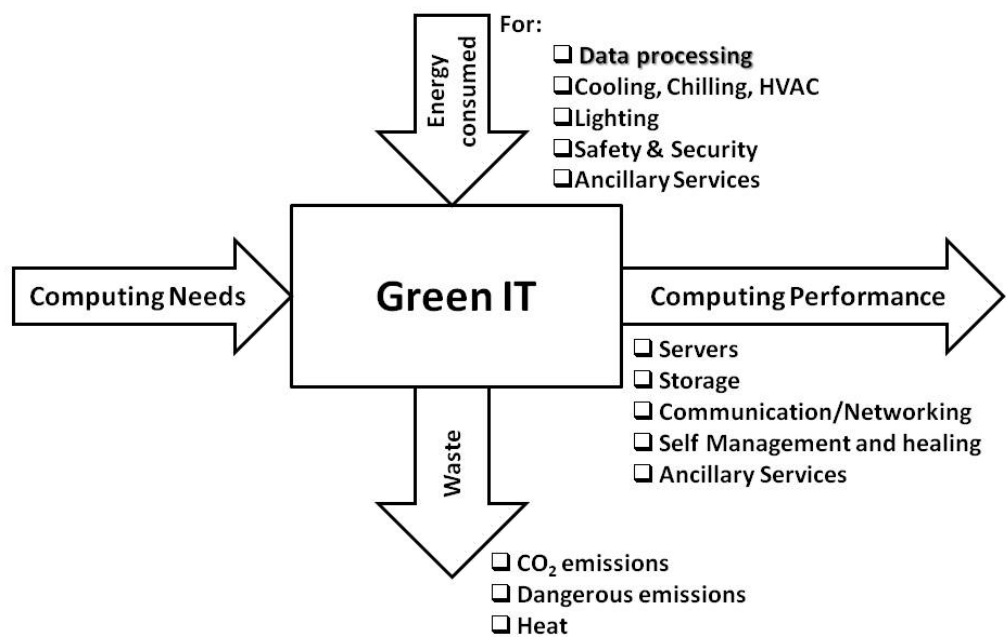

Fig. 10. Role played by Green IT technologies and the functional inferences

In order to characterize the energy efficiency of the data center have been proposed several indexes, such as: power usage effectiveness (PUE), defined as: ratio between total power consumption by data center and the effective power consumption of IT PUE $=\frac{P_{\text {total }}}{P_{I T}}$ and its reciprocal, data center efficiency (DCiE) DCiE $=\frac{P_{I T}}{P_{\text {total }}}($ Avelar, 2010) and also can be defined the data center productivity considered as useful work $(C P)$ divided by energy consumed $\left(W_{c}\right)$ for that $D C P=\frac{C P}{W_{c}}$ (Schulz, 2009). In (Avelar, 2010) a categorization of data center IT subsystems is presented and it can be used as a check list for evaluation the energy efficiency in case of data centers audits. The Green IT optimization problem is a multidimensional one, involving to take in consideration a set of dimensions. From one hand, this includes: infrastructure resource management (IRM) such as: power control and 
power distribution for main data center subsystems such as IT consumption, cooling, chilling, heating, ventilation and air condition, security and safety of power supplying uninterruptible power supplies (UPS), insurance of the secondary reserve-, lighting, avoidance of pollution, scalability design, self healing and highly resilient features, serviceability, hardware granularity, topology and balancing of computation systems in data center rooms, designing of data center components and allowing for them a rapid adaptation in case of updating needs, eco-footprint, mechanical aspects related to the location of IT subsystems and ancillary services. A proposed check-out list for hardware metrics is presented in (Schulz, 2009) (table 5.1). On the other hand, this problem reveals a functionality dimension more related to the software part that endowed the data center. This includes: computation intensification by server virtualization, strategies, for opportunity and based on importance of data process, scheduling of computation tasks and users demands, minimization of data transfer between the data center components -servers, storage arrays -, and avoidance of the waiting and intermediate stages (storage and conversions) in data processing, development of application and data transparent processing from physical computation, storage and communication resources, implementation of a software strategy and granularity of modules toward the safety and recovery possibilities offered for data processed, minimization of data retention stages, optimization of data replication, improving of data rescuing, archiving, and data cataloging. In (Tridib et al., 2009) three different thermal-aware energy-saving schedulers are presented: FCFSBackfill- XInt and FCFS-Backfill-LRH, EDFLRH, and an offline genetic algorithm for SCheduling to minimize thermal cross-INTerference (SCINT), suitable for batch scheduling of backlogs. Related the Green IT energy management systems, an important aspect taken in consideration by the researchers is the ratio cost of energy consumed / calculation efficiency that was above mentioned but with a correction that take into account when this energy is consumed and for what purpose. Thus, it has to be developed with minimum costs systems for optimizing the energy consumption thus to be compliant with the financial aspects of the companies. In this aspect it can be combined intelligent control systems with green energy generation and storage devices.

In present, the energy efficiency of the computing industry and of the IT is a main focus because of the increasing trend related to the power consumption of this segment. For example, data centers, telecommunication switchers and the devices for process control used in industry need to provide more power in order to be compliant with the devices power supply requests. Because the actual IT devices can provide more computation power, they have to be endowed with additional cooling systems which increase the energy consumption. Thus, thermal management became a big problem which has to be solved with maximum efficiency and minimal implementation and supplying costs.

In IT field it can be distinguished two important classes of devices: devices with high reliability (data centers, control devices, telecommunication switchers etc) and auxiliary devices (lights, humidifier and HVAC systems). The high reliability devices cannot be switched off even if there are not currently in use. The second type of devices can be switched off for optimizing the power consumption. In present, many companies made the first step for reducing these costs related to power consumption. They implement a network of peer sensors which automatically switch on the auxiliary loads when they detect the human presence and also, they automatically switch off the auxiliary load if there is no human presence. 
It is known that the HVAC system is an auxiliary load very important for thermal comfort but it is characterized by increased power consumption. For increasing the efficiency and the human comfort, the majority of the auxiliary load (inclusively HVAC systems) can be controlled remote and/or can be programmed.

A high reliability system is a server accessed remote by multiple users, in general using the "desktop" mode such systems can optimize the computation efficiency view as ratio between computation power and energy consumed for that (Gruber \& Keller, 2010). Using the virtual machines the servers can support multiple operating systems offering the possibility for multiple users to work directly on the servers using their specific operating system. To increase the efficiency of the remote computation and to increase the number of the possible users it can be used multiple Virtual Machines (VMware), each of them able to guest different operating systems: Windows, Linux, UNIX, MacOS, thus creating the virtualization of the server. There are 4 major factors that have to be taken in consideration for increasing the server efficiency:

- $\quad$ Safety of power supply providing with direct consequences related to the reliability and computing efficiency and availability

- Promptly served tasks and opportunity of tasks execution, avoiding the unnecessary waiting stages necessary for calculus synchronization by optimal chose of schedulers tools;

- Assuring of optimum environmental conditions for data centers computers by an reliable and intelligent cooling system;

- Data center consolidation taking into account a set of metrics related that reveal the energy efficiency and also the economical and sustainability of computation power inclusive analyzing the Cost/efficiency ratio.

The cooling system of the server is a complex system and its advantages can be taken in consideration if it is endowed with an additional intelligent control for optimizing the temperature level function of costs. For example, the server can be cooled during the night, when the electricity costs are reduced and thus it can function for a couple of hours during day without using the cooling system thus reducing the costs. Also, if the server is cooled adequately, the complex prediction algorithm can anticipate the possibility of over clocking. Also, the prediction algorithm can detect the proper moments for overcharging the server from $20 \%$ (usual value) to more than $60 \%$.

\section{Conclusions}

Nowadays, the energetic processes optimization represents one of the most important and complex research and technological challenges. The impact of these processes is essential for the whole human society and also for the environment. The two essential notions "energy" and "information" are traditionally viewed as dichotomist entities. In fact, these notions are strongly related to both stationary and mobile power sources, in order to reach a highly energy efficiency of the system.

This chapter comes with examples that prove the essential role played by information for driving to optimal and efficient control of power flows on modern systems. Emphasizing the complexity of problems raised by the actual technologies referring to the mobility and computation processes when we search to improve the energy efficiency the structural, 
functional and also several metrics that could be taken into account at the design of modern system are presented toward increasing of the sustainability of these processes.

The problems are provided by the complexity of the systems' structure, with his parameters: sizing of system cells, topology, physical, footprint and economical characteristics. From the functional point of view, are underline the importance of factors such as: the time constants of each "cell" and their aging characteristics, the virtualization as method for intensifying of computation processes, the planning using schedulers or "apriori" programmed control, the valorizing of the bio-inspired methods and paradigms, which are only several functional aspects that was presented. A practical example come to demonstrate the importance of all above mentioned factors when we try to reach the desiderate of the future technological approaches.

\section{References}

Avelar V (2010). Guidence for Calculation of Efficiency (PUE) in Data Centers, White Paper 158, APC Schneider Electric

Bertalanffy L. (1969). General System Theory, Foundations, Development, Applications, George Braziller, New York

Borza P. N. (2007). Electric Power Cell, Patent no. EP1796199

Brandic I. (2009). Towards self-adaptable Cloud services, presentation at EU-COST action IC0804, Toulouse, France

Carp M.C., A.M. Puscas, C. Kertesz, M. Romanca, P. Borza (2010). Monitoring System and Intelligent Control System Used in the Starting Process of a LDH1250 Locomotive, Proceedings of the $12^{\text {th }}$ International Conference on Optimization of Electrical and Electronic Equipment -OPTIM2010, Brasov, Romania ISBN 978-973-131-080-0

Conway B.E. (1999). Electrochemical supercapacitors - scientific fundamentals and technological applications, New York: Kluwer Academic/Plenum Publishers,

EPA (2007). Report to Congress on Server and Data Center Energy Efficiency Public Law 109-431, U.S. Environmental Protection Agency ENERGY STAR Program, August

FENIX (2009). Flexible Electricity Networks to Integrate the expected Energy Evolution - Results, EU FP6 project, http://www.fenix-project.org

Flynn P. F. - chair et al. (2004). Meeting the Energy Needs of Future Warriors, The National Academies Press, 500 Fifth Street, N.W., Washington, DC 20001, USA, ISBN: 0-30953344-9

Gruber R., Vincent Keller (2010). HPC@Green IT Green High Performance Computing Methods, Springer-Verlag Berlin Heidelberg, ISBN 978-3-642-01788-9"

Gualous H., D. Bouquain, A. Berthon and J.M. Kauffmann (2003). Experimental study of supercapacitor serial resistance and capacitance variations with temperature, in Journal of Power Sources, vol. 123, pp. 86-93.

Jiang Z., L. Gao, M. J. Blackwelder and R. A. Dougal (2004). Design and experimental tests of control strategies for active hybrid fuel cell/battery power sources, in Journal of Power Sources, vol. 130, pp. 163-171.

Linden D., T.B. Reddy (2002). Handbook of Batteries, Third Edition, McGraw-Hill Companies, Inc., Two Penn Plaza, New York, NY 10121-2298, USA, ISBN 0-07-135978-8 
Pop V., H. J. Bergveld, D. Danilov, P.L. Regtien, P. H.L. Notten (2008). Battery Management Systems: Accurate State-of-Charge Indication for Battery-Powered Applications, Springer, Germany, ISBN 1402069448.

Rasmussen N. (2010). Power and Cooling Capacity Management for Data Centers, White Paper 150, APC -Schneider Electric

Schulz G (2009). The Green and virtual Data Center, CRC Press Taylor\& Francis Group, Boca Raton FL, ISBN 978-1-4200-8666-9

Sojref D., Borza P. (2008). Comparison of High-Voltage Supercapacitor Approaches and Case Study in Diesel Locomotive Starting System, ESSCAP'08 - 3rd European Symposium on Supercapacitors and Applications, Rome, Italy

Taft D. K., IBM Building Zero-Emission Data Centers, www.eweek.com, 2009

Tridib M., A. Banerjee, G. Varsamopoulos, S. K.S. Gupta, Sanjay Rungta (2009). Spatiotemporal thermal-aware job scheduling to minimize energy consumption in virtualized heterogeneous data centers, Computer Networks, volt 53, pp. 2888-2904, Elsevier 


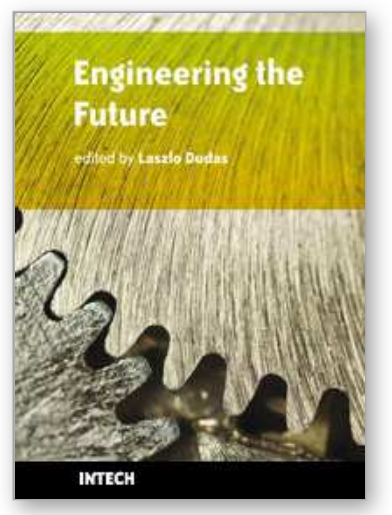

\author{
Engineering the Future \\ Edited by Laszlo Dudas
}

ISBN 978-953-307-210-4

Hard cover, 414 pages

Publisher Sciyo

Published online 02, November, 2010

Published in print edition November, 2010

This book pilots the reader into the future. The first three chapters introduce new materials and material processing methods. Then five chapters present innovative new design directions and solutions. The main section of the book contains ten chapters organized around problems and methods of manufacturing and technology, from cutting process optimisation through maintenance and control to the Digital Factory. The last two chapters deal with information and energy, as the foundations of a prospering economy.

\title{
How to reference
}

In order to correctly reference this scholarly work, feel free to copy and paste the following:

Paul Nicolae Borza, Mihai Sanduleac, Marius Catalin Carp and Ana Maria Puscas (2010). Energy and Information, Engineering the Future, Laszlo Dudas (Ed.), ISBN: 978-953-307-210-4, InTech, Available from: http://www.intechopen.com/books/engineering-the-future/energy-and-information

\section{INTECH}

open science | open minds

\author{
InTech Europe \\ University Campus STeP Ri \\ Slavka Krautzeka 83/A \\ 51000 Rijeka, Croatia \\ Phone: +385 (51) 770447 \\ Fax: +385 (51) 686166 \\ www.intechopen.com
}

\author{
InTech China \\ Unit 405, Office Block, Hotel Equatorial Shanghai \\ No.65, Yan An Road (West), Shanghai, 200040, China \\ 中国上海市延安西路65号上海国际贵都大饭店办公楼405单元 \\ Phone: +86-21-62489820 \\ Fax: +86-21-62489821
}


(C) 2010 The Author(s). Licensee IntechOpen. This chapter is distributed under the terms of the Creative Commons Attribution-NonCommercialShareAlike-3.0 License, which permits use, distribution and reproduction for non-commercial purposes, provided the original is properly cited and derivative works building on this content are distributed under the same license. 